\title{
The Mediating Relationship Between Maladaptive Behaviours, Cognitive Factors, and Generalised Anxiety Disorder Symptoms
}

\author{
Alison E.J. Mahoney,' Megan J. Hobbs,' Alishia D. Williams, ${ }^{2}$ Gavin Andrews' \\ and Jill M. Newby ${ }^{3}$ \\ I Clinical Research Unit for Anxiety and Depression, University of New South Wales at St. Vincent's \\ Hospital, Sydney, New South Wales, Australia \\ ${ }^{2}$ Department of Clinical and Health Psychology, Utrecht University, Utrecht, the Netherlands \\ ${ }^{3}$ Department of Psychology, University of New South Wales, Sydney, New South Wales, Australia
}

\begin{abstract}
Cognitive theories of generalised anxiety disorder (GAD) posit that cognitive and behavioural factors maintain the disorder. This study examined whether avoidance and safety behaviours mediated the relationship between cognitive factors and GAD symptoms. We also examined the reverse mediation model; that is, whether cognitive factors mediated the relationship between maladaptive behaviours and GAD symptoms. Undergraduate psychology students $(N=125$ and $N=292)$ completed the Worry Behaviours Inventory (a recently developed measure of maladaptive behaviours associated with GAD), in addition to measures of intolerance of uncertainty, cognitive avoidance, metacognitive beliefs, and symptoms of GAD and depression. Analyses supported the reliability and validity of the WBI. We consistently found that engagement in maladaptive behaviours significantly mediated the relationship between cognitive factors and symptoms of GAD. The reverse mediation model was also supported. Our results are consistent with the contention that cognitive and behavioural factors contribute to GAD symptom severity.
\end{abstract}

Keywords: generalised anxiety disorder, avoidance, safety behaviour, mediation, cognitive behaviour therapy

The characteristic feature of generalised anxiety disorder (GAD) is excessive and difficult-to-control worry and anxiety about everyday events and activities (American Psychiatric Association [APA], 2013). The Diagnostic and Statistical Manual of Mental Disorders (5th ed.; DSM-5) GAD classification also emphasises the somatic features of the disorder. However, unlike the diagnostic criteria of other anxiety disorders in the DSM-5, maladaptive behaviours are not used to define GAD (APA, 2013; Andrews et al., 2010).

Cognitive models of GAD promote the importance of factors such as intolerance of uncertainty, metacognitive beliefs, and cognitive avoidance in the maintenance of the disorder (Borkovec, Alcaine, \& Behar, 2004; Dugas, Gagnon, Ladouceur, \& Freeston, 1998; Wells, 1999). These factors are thought to directly perpetuate excessive worry and may also increase GAD symptom severity by interacting with maladaptive

Address for correspondence: Alison Mahoney, DPsych (Clin), Clinical Director, Clinical Research Unit for Anxiety and Depression, University of New South Wales at St. Vincent's Hospital, Level 4 O'Brien Centre, 394-404 Victoria Street, Darlinghurst NSW 2010, Australia, 2010. Email: alison.mahoney@svha.org.au

Behaviour Change | Volume 35 | Number 2 | 2018 | pp. 123-138 | (C) The Author(s) 2018

doi $10.1017 /$ bec.2018.13 
behaviours. For instance, Robichaud (2013) explains how beliefs associated with intolerance of uncertainty (e.g., 'A small unforeseen event can spoil everything, even with the best planning') can lead individuals with GAD to engage in unhelpful behaviours, such as excessive planning and procrastinating, in an attempt to reduce or avoid uncertainty associated with everyday situations. Through negative reinforcement, these behaviours are thought to maintain beliefs that uncertainty is aversive and dangerous, and consequently perpetuate excessive worry. Similarly, metacognitive beliefs about worry (e.g., 'worrying could make me go crazy') are thought to prompt individuals with GAD to engage in problematic behaviours in an attempt to prevent the feared consequences of worrying. People may use excessive reassurance and checking in order to stop worrying, and via negative reinforcement, these behaviours can confirm beliefs that worrying is unsafe (Wells, 1995, 1999). Moreover, in an attempt to avoid distressing internal experiences (i.e., thoughts, images, emotions), people with GAD are thought to engage in cognitive avoidance strategies such as thought suppression and worry, as well as avoid situations and activities that trigger the distressing thoughts and images (Sexton \& Dugas, 2008; Wells, 1999). It is likely, therefore, that the cognitive factors implicated in the development and maintenance of GAD are associated with a variety of maladaptive behaviours. It is also possible that a bidirectional relationship between cognitive and behavioural factors exists whereby these factors interact and exacerbate each other and maintain GAD.

Considerable research has examined how cognitive factors contribute to GAD symptom severity (see Andrews, Mahoney, Hobbs, \& Genderson, 2016, for a review). However, the relationship between cognitive factors, maladaptive behaviours and symptoms of GAD has received relatively little empirical scrutiny. Beesdo-Baum et al. (2012) found that higher levels of posttreatment cognitive and behavioural avoidance predicted greater worry at follow-up in their analysis of the effects of two behaviour therapies on GAD symptom severity. Dickson, Ciesla, and Reilly (2012) examined the naturalistic course of cognitive and behavioural avoidance and their relationships with symptoms of anxiety and depression in a sample of adolescents over 7 days. They found that while cognitive avoidance predicted subsequent rumination, worry and sadness, behavioural avoidance was only predictive of subsequent anxiety. However, no studies have examined the associations between maladaptive behaviours and intolerance of uncertainty or metacognitive beliefs, and how these associations relate to GAD symptom severity. Given the potential importance of maladaptive behaviours in the maintenance of GAD, research is needed to demonstrate the associations between these constructs. As such, the primary aim of this study was to examine the mediating relationship between cognitive factors (intolerance of uncertainty, cognitive avoidance, and meta-cognitive beliefs), maladaptive behaviours, and symptoms of GAD.

The paucity of research in this area may relate to the lack of psychometrically sound measures of maladaptive behaviours that are associated with GAD. A self-report measure of these behaviours has recently been developed and evaluated (Mahoney et al., 2016). The Worry Behaviours Inventory (WBI) is a brief index of clinically meaningful, maladaptive behaviours that are associated with GAD symptom severity. The psychometric properties of the measure have been examined in treatment-seeking samples characterised by high rates of probable anxiety and depressive disorders (Mahoney et al., 2016; Mahoney, Hobbs, Newby, Williams, \& Andrews, 2018a, 2018b). The measure assesses two correlated factors: Safety Behaviours (e.g., items assessing checking, watching, planning, reassurance-seeking, and controlling others) and 
Avoidance (e.g., items assessing avoidance of decision making, worrying situations, people and activities). In clinical samples, evidence of the measure's internal consistency, temporal stability, and construct validity has been reported (Mahoney et al., 2016; Mahoney et al., 2018a). However, additional replication in independent samples is essential for confirming the research base of the WBI. Second, the psychometric properties of the WBI in non-treatment seeking samples characterised by lower rates of clinical diagnosis has not been scrutinised and would complement the extant data. If shown, such data would provide researchers and clinicians with additional confidence when using the WBI to assess maladaptive behaviours across a broad dimension of GAD symptom severity.

Following initial analyses to confirm the reliability and factorial, convergent and divergent validity of the WBI in a non-treatment-seeking sample, this cross-sectional study examined the relationship between key cognitive factors (intolerance of uncertainty, metacognitive beliefs, and cognitive avoidance), maladaptive behaviours (avoidance and safety behaviours), and symptoms of GAD. Cognitive models of GAD emphasise the role of cognitive factors in perpetuating the hallmark feature of GAD: excessive worry. As such, we examined the relationships between cognitive and behavioural factors and excessive worry, as well as GAD symptom severity. We predicted that maladaptive behaviours would mediate the relationship between cognitive factors and symptoms of GAD. We also predicted that the reverse mediation model would be supported and that there would be a positive indirect effect of maladaptive behaviours on GAD symptoms through each cognitive factor.

\section{Methods}

\section{Participants}

Two samples were recruited to examine the reliability and validity of the WBI, as well as the relationship between cognitive and behavioural factors, and symptoms of GAD. The test-retest reliability of the WBI was examined in Sample 1, which comprised 125 undergraduate psychology students who were allocated course credit points for their participation in the study; $79.2 \%$ female; $M(S D)$ age $=19.46(2.64)$ years. Most students described themselves as Caucasian Australian (39.2\%), Chinese (24.0\%), Indian (7.2\%) or Asian (5.6\%); had never married (96.8\%); and reported that their primary occupation was being a student $(86.4 \%)$ or working part time $(12.0 \%)$.

The factor structure, internal consistency, and convergent/divergent validity of the WBI, and the mediation models were examined in Sample 2. The latter included 292 undergraduate psychology students; 71.9\% female; $M(S D)_{\text {age }}=19.90$ (3.73) years. Most students described themselves as Caucasian Australian (37.7\%), Chinese (23.6\%), Asian (7.2\%), or Indian (4.8\%); had never married (90.8\%); and reported that their primary occupation was being a student $(70.5 \%)$ or working part time $(25.3 \%)$. In Sample 2, measures of symptom severity indicated that $21 \%$ of participants met criteria for probable GAD; that is, GAD-7 total score $\geq 10 ; M(S D)=6.00$ (4.73) $\mathrm{Mdn}$ (interquartile range) $=5.00$ (7.00), and $25 \%$ for probable major depressive disorder, PHQ-9 total score $\geq 10, \mathrm{M}(\mathrm{SD})=6.71(5.30) \mathrm{Mdn}$ (interquartile range $)=6.00$ (7.00). Demographic variables did not vary across samples, gender $\chi^{2}(1)=2.99, p=.08$; age: $t(415)=1.19, p=.24$, occupation $\left.\chi^{2}(1)=.32, p=.57\right)$, with the exception that students in Sample 1 were more likely to describe themselves as never having been married as compared to Sample 2, $\chi^{2}(1)=4.65, p=.03$. 


\section{Measures}

Worry Behaviours Inventory (WBI). The WBI is a 10-item, self-report measure that assesses how often respondents typically use maladaptive behaviours to control or prevent worry about everyday concerns (Mahoney et al., 2016). Previous studies have shown that two factors explain the co-occurrence of these maladaptive behaviours: Safety Behaviours and Avoidance. Evidence of internal consistency (total $\alpha=.86$; Safety Behaviours subscale $\alpha=.85$; Avoidance subscale $\alpha=.75$ ), temporal stability (total $r=.89$; Safety Behaviours subscale $r=.89$; Avoidance subscale $r=.74$ over 2-4 weeks) and validity (e.g., convergent/divergent, discriminant, and incremental validity) has been demonstrated in treatment-seeking samples (Mahoney et al., 2016, 2018a). Current internal consistency is reported below.

Generalised Anxiety Disorder 7-item (GAD-7). The GAD-7 is a seven-item, selfreport measure of GAD symptom severity experienced in the past 2 weeks (Spitzer, Kroenke, Williams, \& Löwe, 2006). Participants reported how often they had experienced symptoms as either not at all, on several days, on more than half the days, or on nearly every day. A total score of $\geq 10$ indicates a probable GAD diagnosis (sensitivity $=89 \%$ and specificity $=82 \%$; Spitzer et al., 2006). Studies support a one-dimensional structure and provide evidence of internal consistency $(\alpha=.92)$, temporal stability $(r=.83)$, convergent/divergent validity (e.g., correlations with the measures of anxiety and depression), and criterion validity (e.g., sensitivity/specificity with respect to diagnosis; Löwe et al., 2008; Spitzer et al., 2006). Current internal consistency was $\alpha=.89$.

Penn State Worry Questionnaire (PSWQ). The PSWQ is a 16-item measure of trait worry with sound internal consistency $(\alpha=.93)$ and temporal stability $(r=.92$ over 8-10 weeks, Meyer, Miller, Metzger, \& Borkovec, 1990). Factor analyses support a one-dimensional structure and evidence of convergent/divergent validity (via correlations with measures of worry, tension, anxiety, depression) and discriminant validity (e.g., distinguishes between GAD and other anxiety disorders) has been reported (Brown, Antony, \& Barlow, 1992; van Rijsoort, Emmelkamp, \& Vervaeke, 1999). Current internal consistency was PSWQ $\alpha=.83$.

Patient Health Questionnaire-9 (PHQ-9). The PHQ-9 is a nine-item, self-report measure of MDD symptom severity experienced in the past 2 weeks (Kroenke, Spitzer, $\&$ Williams, 2001). Patients reported how often they experienced symptoms as either not at all, on several days, on more than half the days, or on nearly every day with a total score of $\geq 10$ indicative of a probable diagnosis of MDD (Kroenke et al., 2001). Factor analyses support a one or two-factor structure, and evidence of internal consistency ( $\alpha=.86)$, temporal stability ( $r=.84$ over 48 hours), convergent/divergent validity (e.g., correlations with measures of depression and substance use), and criterion validity (e.g., sensitivity/specificity with respect to diagnosis) has been reported (Beard, 126 Hsu, Rifkin, Busch, \& Björgvinsson, 2016; Kroenke et al., 2001; Kroenke, Spitzer, Williams, \& Löwe, 2010). Current internal consistency was $\alpha=.87$.

Intolerance of Uncertainty Scale-12 (IUS-12). The 12-item version of the IUS indexes negative beliefs about and reactions to uncertainty (Carleton, Norton, \& Asmundson, 2007). Participants rate how characteristic each item is of themselves along a 5 -point scale (where $1=$ not at all characteristic of me, $3=$ somewhat characteristic, and $5=$ entirely characteristic of me). The IUS-12 comprises two related subscales: 
Prospective anxiety (anxiety in anticipation of uncertainty; e.g., 'I always want to know what the future has in store for me') and inhibitory anxiety (inhibition of action or experience; e.g., 'When it's time to act, uncertainty paralyses me'). Evidence supporting the structure, internal consistency ( $\alpha=.93$ total score), convergent validity and discriminant validity of the IUS-12 have been reported (McEvoy \& Mahoney, 2011). Current internal consistency was $\alpha=.92$.

Meta-Cognitions Questionnire-30 (MCQ-30). The MCQ-30 is a 30-item, self-report measure of five domains of positive and negative metacognitive beliefs, metacognitive monitoring, and judgments of cognitive confidence (Wells \& Cartwright-Hatton, 2004). Participants rated how much they agreed with each belief (where $1=$ do not agree, 2 = agree slightly, $3=$ agree moderately, and $4=$ agree very much). The five MCQ-30 subscales are: (1) positive beliefs about worry, (2) negative beliefs about uncontrollability and danger, (3) cognitive confidence (assessing confidence in attention and memory), (4) negative beliefs concerning the consequences of not controlling thoughts, and (5) cognitive self-consciousness (the tendency to focus attention on thought processes). Evidence of factorial stability, internal consistency $(\alpha=.93)$, temporal stability $(r=.75$ over 3-16 weeks) and convergent validity has been demonstrated (Wells \& Cartwright-Hatton, 2004). Current internal consistency was $\alpha=.92$.

Cognitive Avoidance Questionnaire (CAQ). The CAQ is a 25-item, self-report measure that assesses the use of five cognitive avoidance strategies in response to threatening thoughts (Sexton \& Dugas, 2008). Participants rated how typical each item is of them on a 5-point scale (where $1=$ not at all typical, $2=$ a little typical, $3=$ somewhat typical, $4=$ very typical and $5=$ completely typical). Subscales measure the strategies of: (1) thought substitution, (2) transformation of images into verbal thoughts, (3) distraction, (4) avoidance of stimuli that trigger unpleasant thoughts, and (5) thought suppression. For the English version of the CAQ, evidence of factorial stability, internal consistency $(\alpha=.95)$, test-retest reliability $(r=.85)$, and convergent/divergent validity has been provided (Sexton \& Dugas, 2008). Internal consistency in the current study was $\alpha=.96$.

\section{Procedure}

To estimate the temporal stability of the WBI in a non-treatment-seeking sample, participants in Sample 1 completed the WBI at Time 1 and then completed the WBI again 2-3 weeks later (Time 2). To investigate the structure, internal consistency and convergent/divergent validity of the WBI, participants in Sample 2 completed the WBI, GAD-7, and PHQ-9. Additionally, these participants completed the IUS12, MCQ-30, CAQ, and PSWQ, such that the relationship between cognitive and behavioural factors and symptoms of GAD could be examined. All data were collected via Qualtrics online survey platform. All participants provided electronic informed consent and could withdraw their participation at any time without consequence or reason. Both projects received ethical approval by the University of New South Wales Human Research Ethics Advisory Panel (\#2730).

\section{Data Analytic Strategy}

Data analysis was conducted in four phases. First, the test-retest reliability of the WBI was examined in Sample 1. Second, the factor structure, internal consistency, 


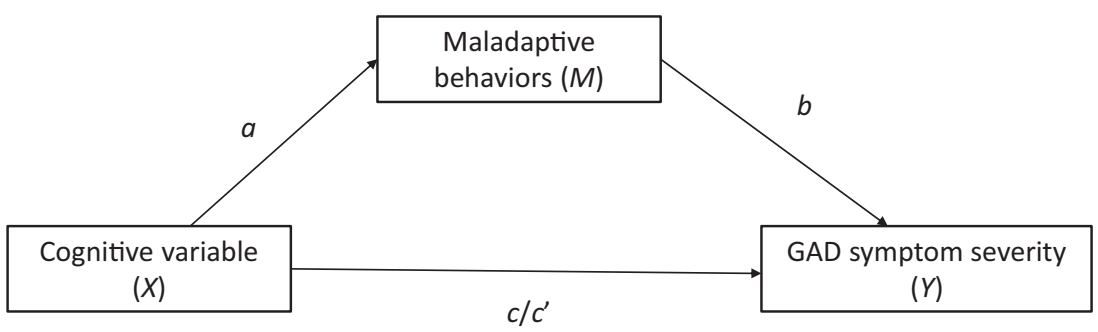

\section{FIGURE 1}

Conceptual mediation model.

Note: $X=$ the proposed causal antecedent: a cognitive variable (metacognitive beliefs [MCQ], cognitive avoidance [CAO] or intolerance of uncertainty [IUS-12]); $Y=$ outcome variable: symptoms of GAD (GAD-7 or PSWQ); $M=$ mediator variable: maladaptive behaviors (WBI); $a=$ effect of $X$ on $M ; b=$ effect of $M$ on $Y ; c^{\prime}=$ direct effect of $X$ on $Y$ controlling $M ; a b=$ indirect effect of $X$ on $Y$ though $M ; c=$ total effect of $X$ on $Y$ (direct and indirect effects).

and convergent/divergent validity of the WBI was evaluated in Sample 2. Third, we investigated whether WBI scores mediated the relationships between cognitive factors (IUS-12, MCQ-30, and CAQ scores) and symptoms of GAD (GAD-7 and PSWQ). We then examined the reverse mediation models as to whether cognitive factors (IUS-12, MCQ-30, and CAQ scores) mediated the relationship between WBI scores and symptoms of GAD (GAD-7 and PSWQ). All analyses were conducted using SPSS version 24 unless otherwise stated.

Test-retest reliability of the WBI. Pearson correlation coefficients were used to assess the temporal stability of the WBI between Times 1 and 2 .

Factor structure and internal consistency of the WBI. The relative fit of the twofactor model of the WBI was estimated using a weighted least squares mean and variance estimator in the MPlus v5.12 software package (Muthén \& Muthén, 1998-2009). Model fit was assessed with reference to the comparative fit index (CFI), Tucker-Lewis index (TLI), and the root mean square error of approximation (RMSEA). Hu and Bentler (1998) suggest that CFI and TLI values $>.90$ indicate acceptable fit and values $\geq .95$ indicate good fit, while RMSEA values $<.05$ indicate good fit. MacCallum, Browne, and Sugawara (1996) recommend that RMSEA values between .08 and .10 indicate mediocre fit, and Browne and Cudeck (1993) propose that RMSEA values $>.10$ indicate poor fit. Consistent with previous evaluations, the internal consistency of the WBI was estimated using Cronbach's alpha.

Convergent and divergent validity of the WBI. Pearson correlation coefficients were used to estimate the association between WBI scores and each of the cognitive factors, GAD symptom severity and depression symptom severity.

Mediation analyses. PROCESS was used to estimate the indirect effect of cognitive variables (the predictor variables, $X$ ) on symptoms of GAD (the outcome variables, $Y$ ) through maladaptive behaviours (the mediator variable, M; see Figure 1) (Hayes, 2012; 


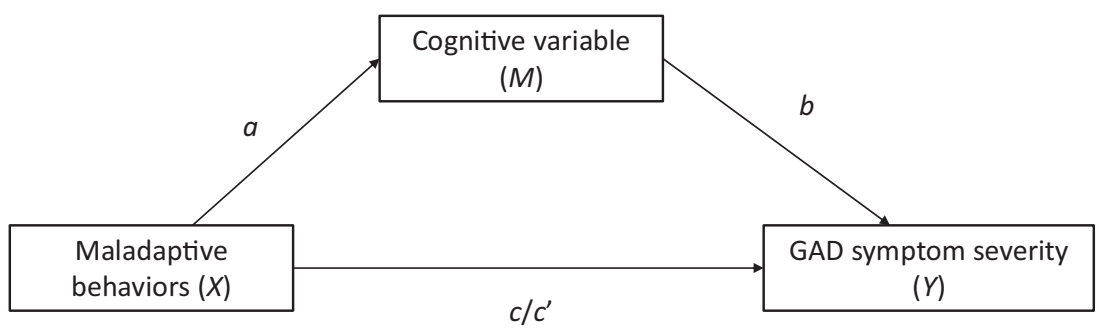

\section{FIGURE 2}

Note: Conceptual mediation model. $X=$ the proposed causal antecedent: maladaptive behaviors (WBI); $Y=$ outcome variable: symptoms of GAD (GAD-7 or PSWQ); $M=$ mediator variable: a cognitive variable (metacognitive beliefs [MCQ], cognitive avoidance [CAO] or intolerance of uncertainty [IUS-12]); $a=$ effect of $X$ on $M ; b=$ effect of $M$ on $Y ; c^{\prime}=$ direct effect of $X$ on $Y$ controlling $\mathrm{M} ; a b=$ indirect effect of $X$ on $Y$ though $M ; c=$ total effect of $X$ on $Y$ (direct and indirect effects).

Hayes \& Rockwood, 2017). The effect of each cognitive variable (i.e., intolerance of uncertainty, metacognitive beliefs, and cognitive avoidance) on GAD symptoms was examined in separate models. We operationalised GAD symptoms by employing the GAD-7 as well as the PSWQ, which indexes the hallmark feature of GAD excessive worry. Reverse mediation models were also evaluated where the effect of maladaptive behaviours (WBI scores, the predictor variables) on symptoms of GAD (the outcome variables) through each cognitive variable (the mediator variables) was estimated (see Figure 2).

Unlike the causal steps approach (Baron \& Kenny, 1986), current recommendations concerning mediation do not require the $a, b$ or even $c$ paths to be significant; mediation is determined by whether the $a b$ path is different from zero (Hayes $\&$ Rockwood, 2017). Bootstrapping is a non-parametric resampling method that generates an estimate of the indirect effect and does not require assumptions about the shape of the sampling distribution that underlie the Sobel test. In bootstrapping analysis, the most stringent test of an indirect effect is if the $95 \%$ bias corrected and accelerated confidence intervals (CIs) for the indirect effect do not include the value of zero. When zero is outside of the $95 \% \mathrm{CI}$, the indirect effect is declared statistically different from zero at $p<.05$ (two-tailed; Hayes \& Rockwood, 2017). In this study, we estimated 5,000 bias-corrected bootstrap 95\% confidence intervals using PROCESS for SPSS. The indirect effect was interpreted by considering the sign $(+/-)$ of the indirect effect, as well as the sign of its constituent components (i.e., the $a$ and $b$ paths). The magnitude of the indirect effects was estimated via the completely standardised indirect effect $\left(a b_{c s}\right)$ statistic because ratio indices and kappa-squared are not currently preferred (Hayes \& Rockwood, 2017; Preacher \& Kelley, 2011; Wan \& Fan, 2015). In this study, the $a b_{c s}$ statistic represents the number of standard deviations (SD) that GAD-7 or PSWQ total scores (the $Y$ variable) increase for each 1 SD increase in the cognitive variables (the IUS-12, MCQ-30, CAQ; $X$ variables) through the WBI (the $M$ variable) (and vice versa for the reverse mediation models). Indirect effects of .01, .09, and .25 were considered small, medium, and large respectively (Kenny, 2016). 


\section{Results}

\section{Preliminary Analyses}

Test-retest reliability of the WBI. In Sample 1, correlations between Time 1 and 2 WBI scores provided evidence of temporal stability $(r=.82, .81$ and .69 for WBI total, Safety Behaviours and Avoidance respectively, all ps <.001). WBI scores did not significantly differ from Time 1 to Time 2 for the WBI total and Safety Behaviours scale: WBI total $M(S D)=16.97(7.93)$ versus $17.22(7.55), t(124)=.73, p=.47$; Safety Behaviours M(SD) $=11.54$ (5.80) versus $11.91(5.57), t(124)=-1.19, p=.24)$; but a small reduction in Avoidance scale scores was observed, $M(S D)=5.79$ (2.41) versus $5.36(2.29), t(124)=2.43, p=.02, d=.17)$.

Factor structure and internal consistency of the WBI. The two-factor structure of the WBI provided a good fit to the current data $(\mathrm{CFI}=.98, \mathrm{TLI}=.97$ and RMSEA $=.07)$. The WBI also yielded good internal reliability estimates $(\alpha=.90, .87$, and .79 for the total, Safety Behaviours and Avoidance subscales, respectively).

Convergent and divergent validity of the WBI. Bivariate correlations for measures of maladaptive behaviours, cognitive constructs and symptoms are presented in Table 1. WBI scores were moderately correlated with all cognitive factors as well as with GAD and depression symptom severity (all ps $<.001$ ). Supporting the divergent validity of the scale, WBI total scores were more strongly correlated with GAD-7 scores than PHQ-9 scores $(z=2.09, p<.05)$.

\section{Mediation Analyses}

Intolerance of uncertainty. First, we examined whether the WBI mediated the relationship between the IUS-12 and the GAD-7 total scores. We found a significant, medium-sized indirect effect of intolerance of uncertainty on GAD-7 scores through the WBI (see Table 2). This pattern of results was replicated when the PSWQ was used as the outcome variable (see Table 2). Second, we examined the reverse mediation model: whether IUS-12 scores mediated the relationship between WBI scores and GAD symptoms. We found a significant medium-sized indirect effect of the WBI on GAD-7 scores through intolerance of uncertainty, which was replicated when the PSWQ was used as the outcome variable (see Table 3). When models were rerun with WBI subscales (i.e., instead of the WBI total score), all results were replicated.

Metacognitive beliefs. First, we examined whether the WBI mediated the relationship between the MCQ-30 and symptoms of GAD. We found significant medium-sized indirect effects of MCQ-30 scores on GAD-7 and PSWQ scores through the WBI (see Table 2). Again, when we estimated the reverse mediation model, we found significant medium-sized indirect effects of the WBI on GAD-7 and PSWQ scores through the MCQ-30 (see Table 3). This pattern of results was observed when WBI subscales replaced WBI total scores in these analyses.

Cognitive avoidance. As expected, we observed significant medium-sized indirect effects of CAQ scores on GAD-7 and PSWQ scores through the WBI (see Table 2). We also found significant medium-sized indirect effects of the WBI on GAD-7 and PSWQ scores through the CAQ (see Table 3). As before, all findings were replicated when WBI subscales were utilized in these mediation models (instead of WBI total scores). 


\section{TABLE 1}

Bivariate Correlations between Measures of Maladaptive Behaviours, Cognitive Factors, and Symptoms of GAD and Depression

\begin{tabular}{|c|c|c|c|c|c|c|c|c|c|}
\hline & $\begin{array}{l}\text { WBI } \\
\text { total }\end{array}$ & $\begin{array}{l}\text { WBI Safety } \\
\text { Behaviours }\end{array}$ & $\begin{array}{l}\text { WBI } \\
\text { Avoidance }\end{array}$ & IUS & MCQ-30 & CAO & GAD-7 & PSWO & PHQ-9 \\
\hline WBI Total & 1 & & & & & & & & \\
\hline WBI Safety Behaviours & .96 & 1 & & & & & & & \\
\hline WBI Avoidance & .79 & .59 & 1 & & & & & & \\
\hline IUS-12 & .64 & .60 & .52 & 1 & & & & & \\
\hline MCQ-30 & .54 & .53 & .41 & .62 & 1 & & & & \\
\hline CAO & .66 & .61 & .58 & .59 & .63 & 1 & & & \\
\hline GAD-7 & .47 & .42 & .43 & .50 & .52 & .51 & 1 & & \\
\hline PSWO & .57 & .53 & .47 & .60 & .52 & .50 & .65 & 1 & \\
\hline PHO-9 & .39 & .32 & .42 & .47 & .48 & .49 & .73 & .49 & 1 \\
\hline
\end{tabular}

Note: All correlations are significant at $p<.001$ WBI $=$ Worry Behaviours Inventory: WBI Safety Behaviours $=$ Worry Behaviours Inventory Safety Behaviours subscale; WBI Avoidance = Worry Behaviours Inventory Avoidance subscale; IUS-12 = Intolerance of Uncertainty Scale - 12-item version; $\mathrm{MCQ}-30$ = Meta-Cognitions Questionnire-30; CAQ = Cognitive Avoidance Questionnaire; GAD-7 = Generalised Anxiety Disorder 7-item Scale; PSWO = Penn State Worry Questionnaire; PHQ9 = Patient Health

Questionnaire-9. 
TABLE 2

Mediation Models where Maladaptive Behaviours Mediate Relationships Between Cognitive Variables and Symptoms of GAD

\begin{tabular}{llllllllll}
\hline $\begin{array}{l}\text { Outcome } \\
\text { variable }(Y)\end{array}$ & $\begin{array}{l}\text { Mediator } \\
\text { variable }(M)\end{array}$ & $\begin{array}{l}\text { Independent } \\
\text { variable }(X)\end{array}$ & $a$ & $b$ & $c^{\prime}$ & $c$ & $a b$ & & $a b_{\mathrm{cs}}$ \\
\hline GAD-7 & WBI & IUS-12 & $.48^{* * *}$ & $.15^{* * *}$ & $.16^{* * *}$ & $.23^{* * *}$ & $.07[.03, .11]$ & $.16[.07, .24]$ \\
& & MCQ-30 & $.26^{* * *}$ & $.16^{* * *}$ & $.11^{* * *}$ & $.15^{* * *}$ & $.04[.02, .06]$ & $.14[.08, .22]$ \\
& & CAO & $.24^{* * *}$ & $.14^{* * *}$ & $.08^{* * *}$ & $.11^{* * *}$ & $.03[.02, .06]$ & $.15[.07, .25]$ \\
PSWO & WBI & IU-12 & $.48^{* * *}$ & $.52^{* * *}$ & $.51^{* * *}$ & $.76^{* * *}$ & $.25[.15, .36]$ & $.20[.12, .28]$ \\
& & MCQ-30 & $.26^{* * *}$ & $.68^{* * *}$ & $.25^{* * *}$ & $.43^{* * *}$ & $.18[.12, .25)]$ & $.22[.15, .30)$ \\
& & CAO & $.24^{* * *}$ & $.70^{* * *}$ & $.14^{* *}$ & $.43^{* * *}$ & $.17[.11, .23]$ & $.27[.18, .37]$ \\
\hline
\end{tabular}

Note: 95\% confidence intervals shown in square brackets. GAD-7 = Generalised Anxiety Disorder 7-item Scale;

PSWO $=$ Penn State Worry Questionnaire; IUS-12 = Intolerance of Uncertainty Scale -12 -item version;

$\mathrm{MCQ}-30=$ Meta-Cognitions Questionnaire-30; CAQ = Cognitive Avoidance Questionnaire; $a=$ effect of $X$ on $M ; b=$ effect of $M$ on $Y ; c^{\prime}=$ effect of $X$ on $Y$ controlling $M$ (direct effect); $c=$ total effect on $Y ; a b=$ indirect effect of $X$ on $Y$ through $M$;

$a b_{\mathrm{cs}}=$ completely standardised indirect effect.

${ }^{* * *} p<.001,{ }^{* *} p<.01$. 


\section{TABLE 3}

Mediation Models where Cognitive Variables Mediate Relationships Between Maladaptive Behaviours and Symptoms of GAD

\begin{tabular}{|c|c|c|c|c|c|c|c|c|}
\hline $\begin{array}{l}\text { Outcome } \\
\text { variable (Y) }\end{array}$ & $\begin{array}{l}\text { Independent } \\
\text { variable }(X)\end{array}$ & $\begin{array}{l}\text { Mediator } \\
\text { variable }(M)\end{array}$ & $a$ & $b$ & $c^{\prime}$ & $c$ & $a b$ & $a b_{\mathrm{cs}}$ \\
\hline \multirow[t]{3}{*}{ GAD-7 } & \multirow[t]{3}{*}{ WBI } & IUS-12 & $.84^{* * *}$ & $.16^{* * *}$ & $.15^{* * *}$ & $.28^{* * *}$ & $.13[.08, .20]$ & $.22[.12, .32]$ \\
\hline & & MCQ-30 & $1.11^{* * *}$ & $.11^{* * *}$ & $.16^{* * *}$ & $.28^{* * *}$ & $.13[.09, .17]$ & $.21[.14, .28]$ \\
\hline & & CAO & $1.80^{* * *}$ & $.08^{* * *}$ & $.14^{* * *}$ & $.28^{* * *}$ & $.14[.09, .20]$ & $.24[.14, .33]$ \\
\hline \multirow[t]{3}{*}{ PSWQ } & \multirow[t]{3}{*}{ WBI } & IU-12 & $.84^{* * *}$ & $.51^{* * *}$ & $.53^{* * *}$ & $.95^{* * *}$ & $.43[.30, .57]$ & $.25[.17, .33]$ \\
\hline & & MCQ-30 & $1.11^{* * *}$ & $.25^{* * *}$ & $.68^{* * *}$ & $.95^{* * *}$ & $.27[.18, .39]$ & $.16[.11, .23]$ \\
\hline & & CAO & $1.80^{* * *}$ & $.14^{* * *}$ & $.69^{* * *}$ & $.95^{* * *}$ & $.25[.11, .41]$ & $.15[.06, .24]$ \\
\hline
\end{tabular}

Note: $95 \%$ confidence intervals shown in square brackets. GAD-7 = Generalized Anxiety Disorder 7-item Scale; PSWQ = Penn State Worry Questionnaire; IUS-12 = Intolerance of Uncertainty Scale -12 -item version; MCQ-30 = Meta-Cognitions

Questionnaire-30; $\mathrm{CAQ}=$ Cognitive Avoidance Questionnaire; $a=$ effect of $X$ on $M ; b=$ effect of $M$ on $Y ; c^{\prime}=$ effect of $X$ on $Y$

controlling $M$ (direct effect); $c=$ total effect on $Y ; a b=$ indirect effect of $X$ on $Y$ through $M ; a b_{c s}=$ completely standardised indirect effect.

$* * * p<.001$. 


\section{Discussion}

This is the first study to demonstrate that maladaptive behaviours mediate the relationship between key cognitive factors (cognitive avoidance, metacognitive beliefs, and intolerance of uncertainty) and symptoms of GAD. Although multiple theories of GAD implicate cognitive and behavioural factors in the maintenance of the disorder, little empirical data has been available to scrutinise the associations between cognitive and behavioural factors and GAD symptoms. These data therefore have important implications for the assessment and theoretical conceptualisation of GAD.

The limited amount of study in this area may in part relate to the lack of a validated measure of maladaptive behaviours associated with GAD. Such a measure has recently been developed and evaluated in treatment-seeking samples (the Worry Behaviours Inventory; Mahoney et al., 2016, 2018a). In the current sample, we found that the two-factor structure of the WBI was confirmed, with both factors demonstrating satisfactory internal stability. The scales also demonstrated adequate temporal reliability. Although we found a significant reduction in WBI Avoidance scores across time, this effect was small $(d=.17)$ and unlikely to be clinically meaningful. We also found evidence to support the convergent validity of the WBI, with total and subscale scores positively correlating with GAD symptom severity and with the cognitive factors proposed to maintain excessive worry. WBI scores were more strongly related to GAD symptom severity than MDD symptom severity, thereby supporting the divergent validity of the scale. Replication across a broad array of samples is essential when establishing the robust psychometric properties of an assessment measure. Current data complements existing psychometric studies of the WBI, which have been conducted almost exclusively in samples reporting high rates of probable anxiety and/or depressive disorders (Mahoney et al., 2016, 2018a). These validation studies support the use of the WBI in samples reporting an extensive range of GAD symptom severities.

The central hypothesis of this study was that maladaptive behaviours would mediate the relationships between cognitive factors - intolerance of uncertainty, metacognitive beliefs, and cognitive avoidance - and symptoms of GAD. Supporting cognitive theories of GAD, we found consistent evidence of mediation, that is, we observed significant positive indirect effects of all three cognitive factors on GAD symptoms via the engagement in maladaptive behaviours. Indeed, these relationships were robust across the assessment of a broad range of GAD symptoms (i.e., symptoms indexed by the GAD-7) and when assessing the core cognitive feature of GAD (i.e., pathological worry, as assessed by the PSWQ). In each mediation model, direct effects were significant and positive when controlling for the mediation path, indicating that engagement in maladaptive behaviours helped to explain a portion of the relationship between cognitive variables and GAD symptoms. This is consistent with cognitive models of GAD that suggests that multiple factors may interact with cognitive variables to maintain the disorder (e.g., life events, mood state, attachment style; see 134 Behar, Di Marco, Hekler, Mohlman, \& Staples, 2009, for an overview). Although our data were correlational, our findings are consistent with the contention that increasing levels of intolerance of uncertainty, metacognitive beliefs, and cognitive avoidance are associated with greater engagement in maladaptive behaviours, which in turn increase GAD symptom severity.

This study also sought to examine evidence of a bidirectional relationship between cognitive and behavioural factors because cognitive models of GAD propose that the 
interaction of cognitive and behavioural factors contributes to GAD symptoms. We therefore evaluated reverse mediation models to test whether cognitive factors mediated the relationship between maladaptive behaviours and GAD symptoms. Again, we observed consistent evidence of mediation, finding positive medium-sized indirect effects of maladaptive behaviours on GAD symptoms through each cognitive factor. Our findings complement the work of Donegan and Dugas (2012), who demonstrated a bidirectional relationship between the cognitive and somatic features of GAD. Our findings are also consistent with work indicating bidirectional relationships between cognitive and behavioural factors within the context of other emotional disorders (e.g., for obsessive-compulsive disorder; Arntz, Voncken, \& Goosen, 2007; Radomsky, Dugas, Alcolado, \& Lavoie, 2014). While novel and preliminary, our findings provide firm support for the basic relationships between cognitive and behavioural factors, and symptoms of GAD.

Our study used a cross-sectional design that prohibits us from drawing causal conclusions. Although cross-sectional data cannot confirm putative causal effects, correlational studies represent a useful and important first step and do not necessarily preclude mediational analyses (Hayes \& Rockwood, 2017). Nevertheless, our findings are consistent with cognitive models of GAD and are supportive of GAD treatment protocols that aim to reduce maladaptive behaviours in an effort to diminish the impact of unhelpful cognitive maintaining factors and reduce symptoms of GAD. Future experimental and intervention studies would extend the current research and establish whether maladaptive cognitive and behavioural factors cause GAD symptoms, and whether reductions in maladaptive behaviours mediate the effect of CBT on symptoms of GAD. In the interests of concision and clarity, we did not examine specific components of cognitive factors (i.e., subscales of the IUS-12, CAQ, and MCQ-30) in the current mediation models. However, previous studies have shown that components of cognitive factors may be differentially associated with symptoms of GAD (e.g., the prospective anxiety dimension of IU has been shown to be uniquely related to excessive worry, while the inhibitory dimension has not; McEvoy \& Mahoney, 2011, 2012). Moreover, the construct overlap between maladaptive behaviours and cognitive factors may be greater for some components of the cognitive factors than others (e.g., in the CAQ, items assessing avoidance of stimuli that trigger unpleasant thoughts may be more strongly related to maladaptive behaviours than items reflecting thought substitution or thought suppression). Mediation models utilising subscale scores may elucidate the complexity of these relationships. Additionally, the indirect effects we observed in this study were not large, and other mechanisms by which cognitive and behavioural factors may influence GAD symptoms need to be explored. Although our study focused on the key cognitive factors thought to maintain GAD, we studied a limited number of these factors, and we studied them in isolation from each other. While this has enhanced our understanding of the rudimentary associations between cognitive and behavioural factors and symptoms of GAD, future research is needed to examine more complex mediation models comprising multiple antecedent and mediation variables. For instance, in the intolerance of uncertainty model of GAD (Dugas et al., 1998), multiple cognitive factors (IU, poor problem orientation, and cognitive avoidance) are thought to interact with maladaptive behaviours and other variables such as life stress and mood state to cause symptoms of GAD, but these multifaceted relationships are yet to be scrutinised. 


\section{Conclusions}

In conjunction with previous evaluations, our study has found consistent evidence to support the validity and reliability of the WBI for individuals experiencing a broad range of GAD symptom severity. Supporting current cognitive models of GAD, this study found that engaging in maladaptive behaviours mediated the effect of intolerance of uncertainty, cognitive avoidance and meta-cognitive beliefs on excessive worry and other symptoms of GAD. Cognitive factors also mediated the relationship between maladaptive behaviours and symptoms of GAD.

\section{Acknowledgments}

This research received no specific grant from any funding agency, commercial or notfor-profit sectors. AM is supported by an Australian Government Research Training Program Scholarship. JN is supported by a NHMRC Early Career Research Fellowship (1033787).

\section{Declaration of Interest}

None

\section{Ethical Standards}

The authors assert that all procedures contributing to this work comply with the ethical standards of the relevant national and institutional committees on human experimentation and with the Helsinki Declaration of 1975, as revised in 2008.

\section{References}

Andrews, G., Hobbs, M.J., Borkovec, T.D., Beesdo, K., Craske, M.G., Heimberg, R.G., ... Stanley, M.A. (2010). Generalised worry disorder: A review of DSM-IV generalised anxiety disorder and options for DSM-V. Depression and Anxiety, 27, 134-147.

Andrews, G., Mahoney, A.E.J., Hobbs, M.J., \& Genderson, M. (2016). Treatment of generalized anxiety disorder: Therapist guides and patient manual. Oxford, UK: Oxford University Press.

American Psychiatric Association. (2013). Diagnostic and statistical manual for mental disorders (5th ed.). Washington, DC: Author.

Arntz, A., Voncken, M., \& Goosen, A. (2007). Responsibility and obsessive-compulsive disorder: An experimental test. Behavior Research and Therapy, 45, 425-435.

Baron, R.M., \& Kenny, D.A. (1986). The moderator-mediator variable distinction in social psychological research: Conceptual, strategic, and statistical considerations. Journal of Personality and Social Psychology, 51, 1173-1182.

Beard, C., Hsu, K.J., Rifkin, L.S., Busch, A.B., \& Björgvinsson, T. (2016). Validation of the PHQ-9 in a psychiatric sample. Journal of Affective Disorders, 193, 267-273.

Beesdo-Baum, K., Jenjahn, E., Hofler, M., Lueken, U., Becker, E.S., \& Hoyer, J. (2012). Avoidance, safety behavior, and reassurance seeking in generalised anxiety disorder. Depression and Anxiety, 29, 948-957.

Behar, E., Di Marco, I.D., Hekler, E.B., Mohlman, J., \& Staples, A.M. (2009). Current theoretical models of generalized anxiety disorder (GAD): Conceptual review and treatment implications. Journal of Anxiety Disorders, 23, 1011-1023.

Borkovec, T.D., Alcaine, O.M., \& Behar, E. (2004). Avoidance theory of worry and generalised anxiety disorder. In R.G. Heimberg, C.L. Turk \& D. Mennin (Eds.), Generalised anxiety disorder: Advances in research and practice (pp. 77-108). New York, NY: Guilford Press. 
Brown, T.A., Antony, M.M., \& Barlow, D.H. (1992). Psychometric properties of the Penn State Worry Questionnaire in a clinical anxiety disorders sample. Behavior Research and Therapy, 30, 33-37.

Browne, M.W., \& Cudeck, R. (1993). Alternative ways of assessing model fit. In K.A. Bollen \& J.S. Long (Eds.), Testing structural equation models (pp. 136-162). Beverly Hills, CA: Sage.

Carleton, R.N., Norton, P.J., \& Asmundson, G. (2007). Fearing the unknown. A short version of the Intolerance of Uncertainty Scale. Journal of Anxiety Disorders, 21, 105-117.

Dickson, K.S., Ciesla, J.A., \& Reilly, L.C. (2012). Rumination, worry, cognitive avoidance, and behavioral avoidance: Examination of temporal effects. Behavior Therapy, 43, 629640.

Donegan, E., \& Dugas, M.J. (2012). Generalized anxiety disorder: A comparison of symptom change in adults receiving cognitive-behavioral therapy or applied relaxation. Journal of Consulting and Clinical Psychology, 80, 490-496.

Dugas, M.J., Gagnon, F., Ladouceur, R., \& Freeston, M.H. (1998). Generalised anxiety disorder: A preliminary test of a conceptual model. Behavior Research and Therapy, 36, 215-226.

Hayes, A.F. (2012). Process: A versatile computational tool for observed variable mediation, moderation, and conditional process modeling. Unpublished manuscript, The Ohio State University. Retrieved from http://www.afhayes.com/public/process2012.pdf

Hayes, A.F., \& Rockwood, N.J. (2017). Regression-based statistical mediation and moderation analysis in clinical research: Observations, recommendations, and implementation. Behavior Research and Therapy, 98, 39-57.

Hu, L.T. \& Bentler, P.M. (1998). Fit indices in covariance structure modeling: Sensitivity to underparameterized model misspecification. Psychological Methods, 3, 424-453.

Kenny, D.A. (2016, January 15). Effect size of the indirect effect and the computation of power. Retrieved from http://davidakenny.net/ $\mathrm{cm} /$ mediate.htm\#IE

Kroenke, K., Spitzer, R., \& Williams, J.B. (2001). The PHQ-9: Validity of a brief depression severity measure. Journal of General Internal Medicine, 16, 606-613.

Kroenke, K., Spitzer, R., Williams, J.B., \& Löwe, B. (2010). The Patient Health Questionnaire Somatic, Anxiety, and Depressive Symptom Scales: A systematic review. General Hospital Psychiatry, 32, 345-359.

Löwe, B., Decker, O., Müller, S., Brähler, E., Schellberg, D., Herzog, W., \& Herzberg, P.Y. (2008). Validation and standardization of the Generalized Anxiety Disorder Screener (GAD-7) in the general population. Medical Care, 46, 266-274.

MacCallum, R.C., Browne, M.W., \& Sugawara, H.M. (1996). Power analysis and determination of sample size for covariance structure modeling. Psychological Methods, 1, 130-149.

Mahoney, A., Hobbs, M.J., Newby, J.M., Williams, A.D., Sunderland, M. \& Andrews, G. (2016). The Worry Behaviors Inventory: Assessing the behavioral avoidance associated with generalized anxiety disorder. Journal of Affective Disorders, 203, 256-264.

Mahoney, A., Hobbs, M.J., Newby, J.M., Williams, A.D., \& Andrews, G. (2018a). Psychometric properties of the Worry Behaviors Inventory: Replication and extension in a large clinical and community sample. Behavioral and Cognitive Psychotherapy, 46, 84-100.

Mahoney, A., Hobbs, M.J., Newby, J.M., Williams, A.D., \& Andrews, G. (2018b). Maladaptive behaviours associated with generalised anxiety disorder: An item response theory analysis. Behavioural and Cognitive Psychotherapy. Advance online publication.

McEvoy, P.M., \& Mahoney, A. (2011). Achieving certainty about the structure of intolerance of uncertainty in a treatment seeking sample with anxiety and depression. Journal of Anxiety Disorders, 25, 112-122.

McEvoy, P.M., \& Mahoney, A. (2012). To be sure, to be sure: Intolerance of uncertainty mediates symptoms of various anxiety and depressive disorders. Behavior Therapy, 43, 533-545.

Meyer, T.J., Miller, M.L., Metzger, R.L., \& Borkovec, T.D. (1990). Development and validation of the Penn State Worry Questionnaire. Behavior Research and Therapy, 28, 487-495. 
Muthén, L.K. \& Muthén, B.O. (1998-2009). Mplus user's guide. Los Angeles, CA: Muthén \& Muthén.

Preacher, K.J., \& Kelley, K. (2011). Effect size measures for mediation models: Quantitative strategies for communicating indirect effects. Psychological Methods, 16, 93-115.

Radomsky, A.S., Dugas, M.J., Alcolado, G.M., \& Lavoie, S.L. (2014). When more is less: Doubt, repetition, memory, metamemory, and compulsive checking in OCD. Behavior Research and Therapy, 59, 30-39.

Robichaud, M. (2013). Cognitive behavior therapy targeting intolerance of uncertainty: Application to a clinical case of generalised anxiety disorder. Cognitive and Behavioral Practice, 20, 251-263.

Sexton, K.A., \& Dugas, M.J. (2008). The Cognitive Avoidance Questionnaire: Validation of the English translation. Journal of Anxiety Disorders, 22, 355-370.

Spitzer, R.L., Kroenke, K., Williams, J.B., \& Löwe, B. (2006). A brief measure for assessing generalized anxiety disorder: The GAD-7. Archives of Internal Medicine, 166, 1092-1097.

Wan, Z., \& Fan, X. (2015). Monotonicity of effect sizes: Questioning kappa-squared as mediation effect size measure. Psychological Methods, 20, 193-203.

Wells, A. (1995). Meta-cognition and worry: A cognitive model of generalized anxiety disorder. Behavioral and Cognitive Psychotherapy, 23, 301-320.

Wells, A. (1999). A metacognitive model and therapy for generalised anxiety disorder. Clinical Psychology and Psychotherapy, 6, 86-95.

Wells, A., \& Cartwright-Hatton, S. (2004). A short form of the metacognitions questionnaire: Properties of the MCQ-30. Behavior Research and Therapy, 42, 385-396.

van Rijsoort, S., Emmelkamp, P., \& Vervaeke, G. (1999). The Penn State Worry Questionnaire and the Worry Domains Questionnaire: Structure, reliability and validity. Clinical Psychology and Psychotherapy, 6, 297-307. 